\title{
Adding Flexibility to Structure Similarity Queries on XML Data
}

\author{
Paolo Ciaccia and Wilma Penzo \\ DEIS - CSITE-CNR \\ University of Bologna, Italy \\ $\{$ pciaccia, wpenzo\}@deis . unibo.it
}

\begin{abstract}
The presence of structure inside XML documents poses the hard challenge of providing flexible query matching methods for effective retrieval of results. In this paper we present an approach that faces this issue in a twofold fashion: 1) it exploits new approximations on data structure; 2) it provides a relevance ranking method that takes into account the degree of correctness and completeness of results with respect to a given query, as well as the degree of cohesion of data retrieved.
\end{abstract}

\section{Introduction and Motivation}

The increasing availability of large digital libraries is raising XML to be the new standard for data representation. Because of the heterogeneity of document collections, querying blindly XML data requires a great deal of flexibility not to fall into the trap of "empty results", often caused by too strict constraints. In fact, the presence of structure inside XML documents is double-faced: on one hand, it helps to define the context where information has to be retrieved (for instance, "Retrieve the director of the movie entitled Casablanca"), on the other, it may be an obstacle to the retrieval of relevant information that slightly differs in data organization.

For instance, consider a user looking for stores in New York city selling CD's authored by Elton John, and containing songs with "love" in the title. Among the two stores listed in Doc1 of Fig. 1 only the former fully satisfies query requirements. This is because it is the only store in Doc1 that presents a city attribute, thus making condition on "New York" checkable. But, it is evident that also the second store is relevant to the query, and should be returned.

Thus, relaxation on query requirements is currently the scope of many proposals $4,7,101920,21]$. These works deal with similarity queries that approximate results both on semantics and on structural conditions. However, most of the above approaches do not recognize the second CD store of Doc1 as relevant. Actually, except for ApproXQL [17, they do not cope with the problem of providing structurally incomplete results, i.e. results that only partially satisfy query requirements on data organization. Further, although supposing that condition on New York is checkable for the second CD store in Doc1, none of the above 

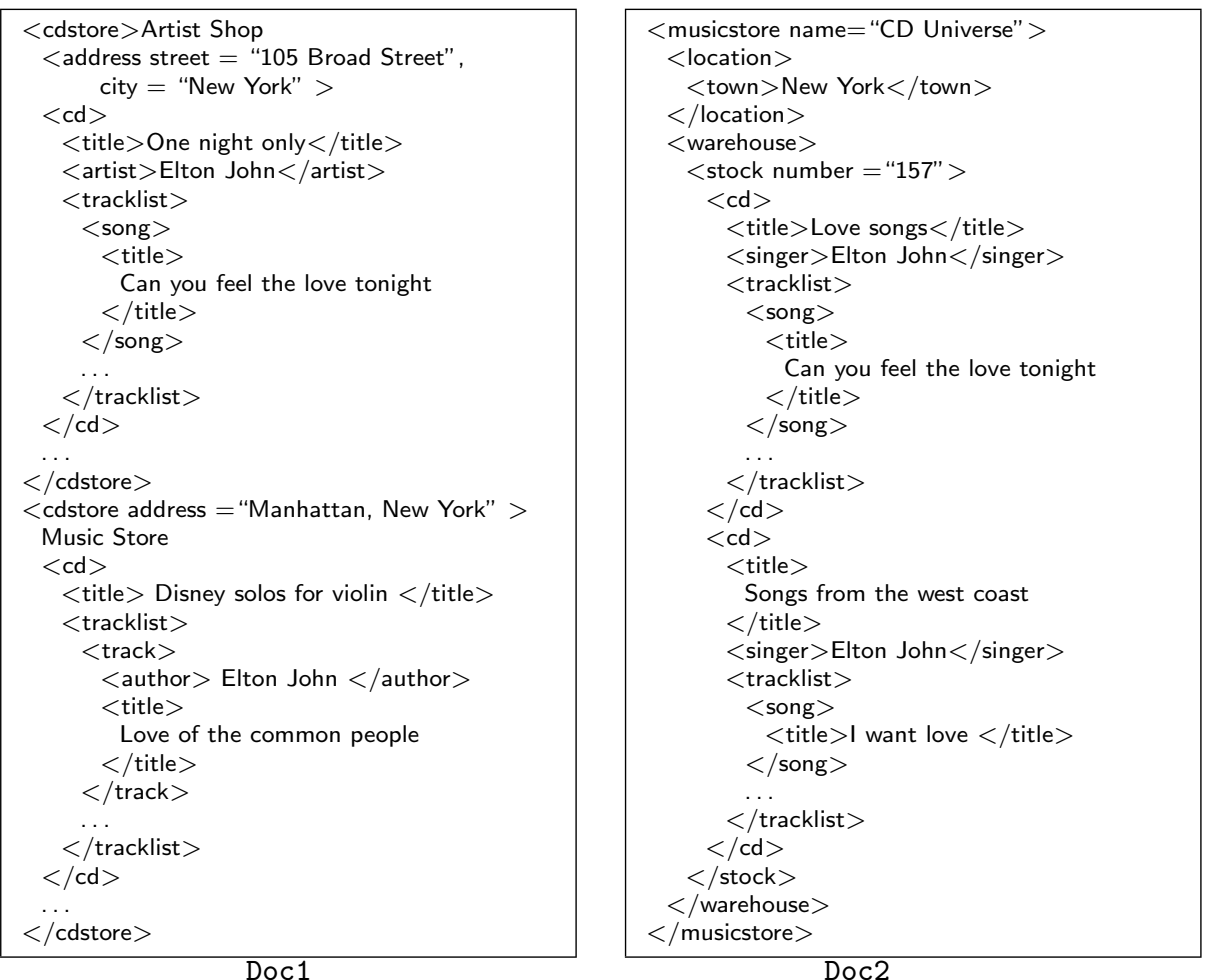

Fig. 1. Sample XML documents

method would recognize it as relevant, because of a slight difference on data organization. In fact, Elton John appears as a track author rather than as the CD author, as specified by the query. This emphasizes the need of more flexibility in the evaluation of structure conditions, most of all in absence of knowledge of data organization.

As to relevance ranking, relaxations on both semantics and structure are expected to affect the score of results. However, in many proposals [4,7,10,20] the use of wildcards flatten the score of the retrieved data, which is considered relevant no matter how much "sparse" it is. Only in ApproXQL the number of elements matching a wildcard contributes to the ranking of results. But none of the above approaches takes into account neither the query rate satisfied by an answer, i.e. the completeness of the answer, as well as its structural correctness, nor the cohesion of results. In fact, also sparseness of data retrieved plays an important role for relevance evaluation. For instance, consider the CD store in Doc2. Although it satisfies the query conditions, note that the required CD's belong to the stock " 157 " of the store's warehouse. This changes the context where information is retrieved and, in this case, this may possibly mean that the 
CD's are not currently available for sale. Then, this store contains two relevant CD's, thus probably it is expected to score higher than others.

In this paper we provide a method to find the approximate embeddings of a user query in XML document collections. Our proposal captures the relaxations described above, and provides a ranking measure that takes into account the degree of correctness and completeness of results with respect to a given query, as well as the degree of cohesion of data retrieved. Our proposal relies on tree representation both for XML documents [3], and for queries. The outline is as follows: In Section 2 we discuss related work. In Section [3] we start from the unordered tree inclusion problem [12] to relate query and data trees through embeddings; this is extended in two directions: 1) to capture also partial matching on query structure, and 2) to assign a score to the retrieved embeddings. In Section 4 we define the SATES (Scored Approximate Tree Embedding Set) function, to retrieve and score embeddings, and we give a flavour of the flexibility and the effectiveness of the method through examples. However, we report its formal definition in appendix. Section [5] is devoted to relevance ranking computation. Properties of correctness and completeness of embeddings are presented. We also compare our method with other related approaches. In Section [6] we briefly discuss implementation and, finally, in Section 7 we conclude and discuss our planned future work.

\section{Related Work}

Several current proposals $46 / 7 / 8$ 10 11/16 17/20|21] deal with similarity queries on XML data. In XXL [20] similarity basically depends on document content, through the evaluation of vague predicates, and information on structure is only exploited to combine semantic similarity scores. Partial match on query structure is not supported. XIRQL [10] introduces the concept of index object to specify document units. Terms are weighted locally to these units, thus defining the relevance of each term in the scope given by the node. Thus, structural conditions act as filters to determine the context where information has to be searched in. Partial match on query structure yet is not discussed. The latter feature is neglected also in [7]. The ranking of scores depends on the context where data is retrieved, and semantic similarity is not exploited.

Then, other approaches [417] adopt tree-based retrieval models to captures XML data relationships in a natural way. In fact, classical models (e.g. vector space, probabilistic) lack the ability to handle structural information, although some extensions have been proposed for them [18|21]. In [4] relevance ranking is based on tree pattern relaxations. Nodes and edges in a query tree are assigned pairs of weights that denote scores for exact and relaxed matching, respectively. Sum is used to combine scores of each single node/edge matching. Partial match on query structure is not supported. Only ApproXQL [17] considers partial match on query structure.

Then, in all above-cited proposals cohesion of data retrieved is not considered for relevance. In [7] the length of paths connecting two matching nodes is 
neglected; In 20. wildcards are intentionally used, thus discarding the (negative) contribution of intermediate nodes between matching data; In [10] this issue is not discussed. In [17] similarity scores depend on the costs of basic transformations applied on the query tree. These costs do not depend on data. Thus, semantic relationships, like synonymy, are not exploited.

Further, to our knowledge, for a given document, all proposed methods return only the best (in some cases, approximate) matching. The set-oriented approach used in the $S A T E S$ function also captures the relevance given by multiple occurrences of the query pattern in the retrieved data.

Other related approaches deal with matching elements of data schemas, usually represented as graphs 1415. In [14] trees are used as a starting point to perform schema matching (for instance, to compare XML documents). Structural similarity is measured as the fraction of leaves appearing in the matching. The notion of completeness is then limited to leaf coverage. Cohesion is not explicitly discussed, although it influences the final similarity of trees, through the use of descreasing factors. In [15] quality of results is measured in terms of the effort the user needs to spend to obtain a perfect match from the automatically generated one. This is accomplished through the addition/deletion of node pairs in the mapping. This suggests a measure of incompleteness of results (when nodes are to be added), but it does not provide any information on cohesion of data retrieved.

\section{Relaxing the Tree Embedding Problem}

In a tree view of documents and queries, several proposals 4 4/7/10/17/20 rely on tree/graph pattern matching techniques to decide if a document $d$ is a possible answer to a query $q$.

In most of these works, all query nodes must have a corresponding matching node in the document tree, and each parent-child relationship should be guaranteed at least by an ancestor-descendant one in the data tree. This is also known as the tree embedding problem [12]. Nevertheless, in many cases these conditions are too restrictive, yet being not flexible enough to effectively deal with the structural heterogeneity of XML documents. In fact, in absence of knowledge of data organization, documents often do not correspond completely to query requirements, yet being mostly relevant although in absence of some conditions. This is the case, for instance of the second CD store of Doc1, that does not present any city element/attribute to be checked for the query condition on New York. Further, frequently, documents do not exactly fit the structural constraints provided in a user query. An example is Doc1, where Elton John appears as track author, instead of CD author as required in the sample query presented above.

Our work basically loosens the strictness of this approach, that often leads to empty results because of minor differences in data organization. The key aspects of our proposal are: 
1. the relaxation on the concept of total embedding of a query tree $t_{q}$ in a document tree $t_{d}$, in that we admit partial structural match of the query tree, as well as approximations on structural relationships; further, the match is relaxed to consider semantic similarity between nodes;

2. ranking of results with respect to the cohesion of data retrieved, to the relaxation of semantic and structural constraints, and to the coverage rate of the query;

3. a set-oriented approach to results, that depending on different relaxations on requirements, identifies possible alternatives that the user may be interested in. This also strengthens the relevance of data presenting multiple occurrences of query patterns.

Point 1) leads to the introduction of our interpretation of approximate tree embedding. First, we report the basic notation we will use throughout paper and appendix. As to tree representation of XML documents, we follow the XML Information Set standard [3] (with root and label as document element and local name properties, respectively, and parent and children as the homonymous properties, denoting a parent-child relationship between argument nodes, and the set of first level children of a given node, respectively). We also define some additional functions for nodes $n, n_{1}$, and $n_{2}$ in a tree: $\operatorname{leaf}(n)$ iff $\operatorname{children}(n)=\emptyset$, and ancestor $\left(n_{1}, n_{2}\right)$ iff $\left(\operatorname{parent}\left(n_{1}, n_{2}\right) \vee \exists n \in N\right.$ s.t. $\left(\operatorname{parent}\left(n_{1}, n\right) \wedge\right.$ ances$\left.\operatorname{tor}\left(n, n_{2}\right)\right)$. We denote with $\mathcal{T}$ the set of such trees. Given a tree $t \in \mathcal{T}$, nodes $(t)$ returns the set of all nodes of $t$. Then, given a node $n$ in a tree $t \in \mathcal{T}$, we define $\operatorname{supp}(n)$ the subtree of $t$ rooted at $n$, and we call it the support of $n$ in $t$. Let $\mathcal{T}_{D} \subset \mathcal{T}$ be the set of all data trees, and $\mathcal{T}_{Q} \subset \mathcal{T}$ be the set of all query trees.

We intentionally neglect the presence of namespaces and links (IDREFs) inside XML documents and we assume data is well-formed, but we do not require validity with respect to a Document Type Definition (DTD).

Definition 1 (Approximate Tree Embedding (ATE)). Given a query tree $t_{q} \in \mathcal{T}_{Q}$ and a document tree $t_{d} \in \mathcal{T}_{D}$, an approximate tree embedding of $t_{q}$ in $t_{d}$ is a partial injective function $\tilde{e}\left[t_{q}, t_{d}\right]: \operatorname{nodes}\left(t_{q}\right) \rightarrow \operatorname{nodes}\left(t_{d}\right)$ such that $\forall q_{i}, q_{j}$ in the domain of $\tilde{e}(\operatorname{dom}(\tilde{e}))$ :

a). $\operatorname{sim}\left(\operatorname{label}\left(q_{i}\right), \operatorname{label}\left(\tilde{e}\left(q_{i}\right)\right)\right)>0$, where $\operatorname{sim}$ is a similarity operator that returns a score normalized in $[0,1]$ stating the semantic similarity between the two given labels

b). $\operatorname{parent}\left(q_{i}, q_{j}\right) \Rightarrow\left(\operatorname{ancestor}\left(\tilde{e}\left(q_{i}\right), \tilde{e}\left(q_{j}\right)\right) \vee \operatorname{sibling}\left(\tilde{e}\left(q_{i}\right), \tilde{e}\left(q_{j}\right)\right)\right)$

c). $\operatorname{sibling}\left(q_{i}, q_{j}\right) \Rightarrow\left(\operatorname{sibling}\left(\tilde{e}\left(q_{i}\right), \tilde{e}\left(q_{j}\right)\right) \vee\right.$ ancestor $\left.\left(\tilde{e}\left(q_{i}\right), \tilde{e}\left(q_{j}\right)\right)\right)$

Let $\mathcal{E}$ be the set of approximate tree embeddings.

Points b) and $c$ ) of Def. 1 capture both the presence of intermediate nodes between $\tilde{e}\left(q_{i}\right)$ and $\tilde{e}\left(q_{j}\right)$ (ancestorship), and additional approximations on structural conditions (sibling relationship), that we call structure unbalances, as shown in the following example:

Example 1. Recall the sample query presented in the Introduction: Retrieve stores in New York city selling CD's authored by Elton John, and containing 


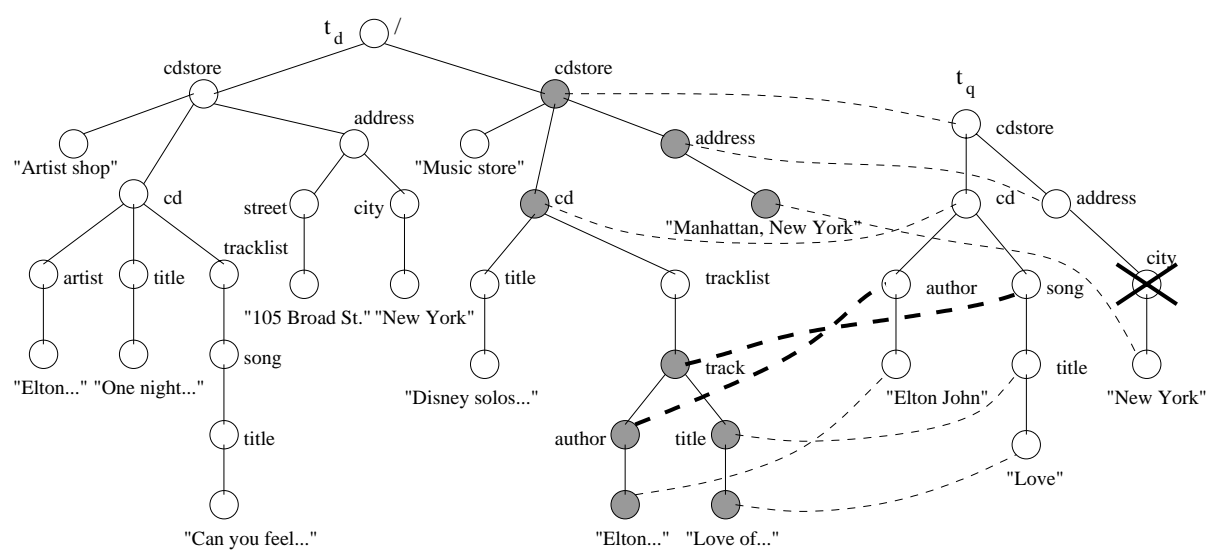

Fig. 2. Partial coverage and unbalance of query tree in Doc1

songs with "love" in the title. Consider Fig. 2 where a possible result is shaded in the Doc1 tree. Note that $t_{q}$ is only partially covered in this solution, since the city node does not have any correspondence in $t_{d}$. Further, the emphasized dashed lines show a structure unbalance.

As to point 2), in order to specify the ranking of results, embeddings are to be assigned a score, according to a relevance ranking function $\rho$.

Because of the flexibility allowed in the retrieval of embeddings, different types of relaxations are to be taken into account for relevance score computation. For instance, partiality of the $\tilde{e}$ function affects the completeness of results. Approximations according to the a) point of Def. 1 influence the semantic correctness of the retrieved data. Relaxations like those allowed in b) and c) denote structural discrepancies as well as low cohesion of data since intermediate nodes are allowed in the document tree. This latest relaxation is evident in Fig. 3 where the shaded solution in the Doc2 tree presents a relevant CD in the stock " 157 " of the store's warehouse.

This denotes fragmentation of data retrieved, that contributes to lower the final result score. Our ranking function takes into account all these features, as detailed below.

Definition 2 (Relevance Ranking Function). We denote with $\rho$ a ranking function that, given a triple $\left(t_{q}, t_{d}, \tilde{e}\left[t_{q}, t_{d}\right]\right)$, returns a score $\sigma \in S=[0,1]$ such that, with respect to the approximate embedding $\tilde{e}$ of the query expressed by $t_{q}$ in the document expressed by $t_{d}, \sigma$ is obtained as a combination of the following components:

- $\gamma_{1}$, that indicates how much $\tilde{e}$ is semantically complete with respect to the given query

- $\gamma_{2}$, which denotes semantic correctness of $\tilde{e}$, in that it states how well the embedding satisfies semantic requirements 


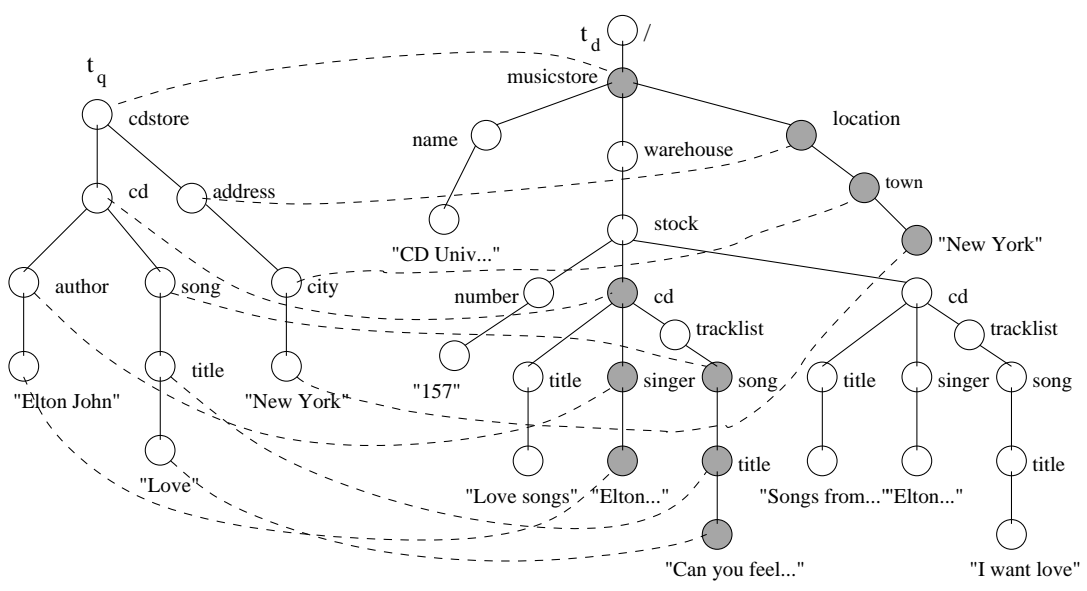

Fig. 3. Partial coverage of query tree and low cohesion in Doc2

- $\gamma_{3}$, that represents the structural completeness of $\tilde{e}$ with respect to the given query; it denotes the structural coverage of the query

$-\gamma_{4}$, that expresses structural correctness of $\tilde{e}$, in that it is a measure of how well constraints on structure are respected in the embedding

$-\gamma_{5}$, which specifies cohesion of $\tilde{e}$, by providing the grade of fragmentation of the retrieved embedding

Thus, $\sigma=\phi\left(\gamma_{1}, \gamma_{2}, \gamma_{3}, \gamma_{4}, \gamma_{5}\right)$, with $\phi$ a combine function, states the overall similarity score of the embedding $\tilde{e}$. Formally:

$$
\rho: \mathcal{T}_{Q} \times \mathcal{T}_{D} \times \mathcal{E} \rightarrow S
$$

As to the components of a score $\sigma$, in Section [5] we will detail each $\gamma_{i}$, when discussing relevance computation. Now we are ready to introduce a scored approximate tree embedding.

Definition 3. (Scored Approximate Tree Embedding) A scored approximate tree embedding $\tilde{e}_{s}$ is an approximate tree embedding extended with a score in $\mathcal{S}$. Formally: $\tilde{e}_{s}: \mathcal{S} \times \mathcal{E}$.

With regard to point 3), consider Fig. 4. The CD store already retrieved through the embedding of Fig. 3 presents a second relevant CD. Thus, we expect this music store to be a high relevant answer to the user query, since requirements on CD occur twice. On the other hand, Doc1 contains two relevant stores, as shown in figures 2 and 5 . This also increases the relevance of this document.

In order to capture these situations, our tree embedding method considers the presence of multiple occurrences of query requirements, by providing a setoriented approach to results. This information is then exploited to strengthen the score of dense results. 


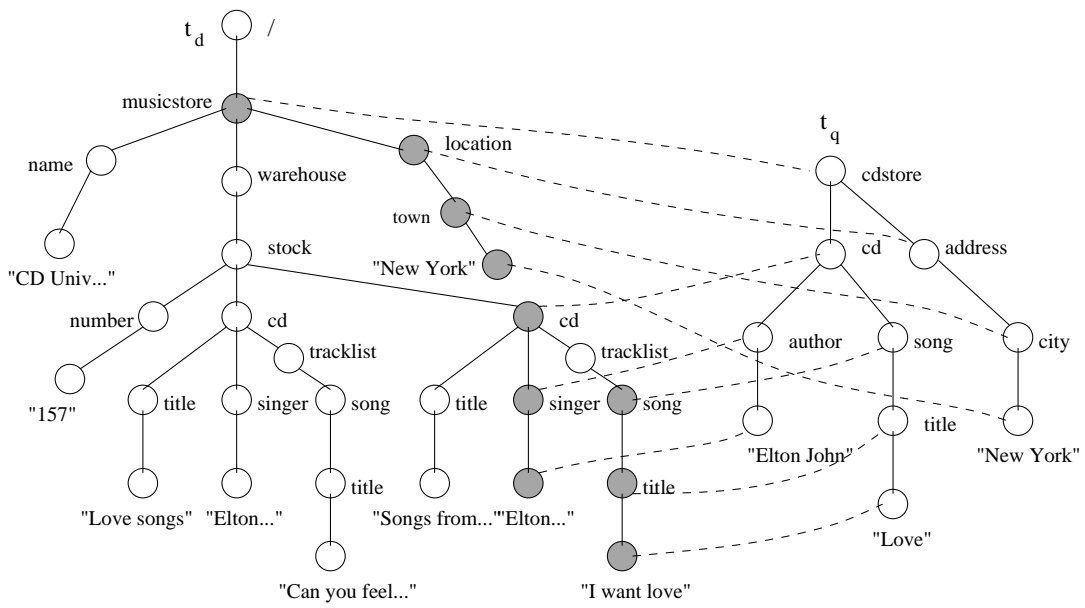

Fig. 4. Further occurrence of query tree in Doc2

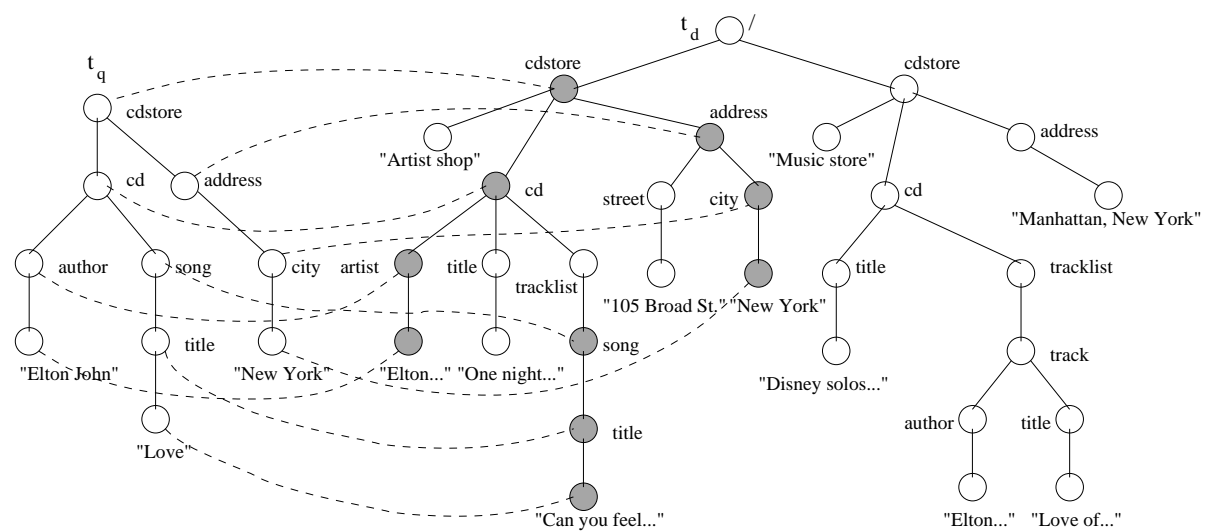

Fig. 5. Full coverage of query tree in Doc1

\section{Approximate Embedding of Query Trees}

The similarity function we propose for retrieval and scoring of embeddings of a query tree in a document tree, is given by the SATES (Scored Approximate Tree Embedding Set) function.

Definition 4 (SATES Function). We define the Scored Approximate Tree Embedding Set Function as:

$$
S A T E S: \mathcal{T}_{Q} \times \mathcal{T}_{D} \rightarrow 2^{\mathcal{S} \times \mathcal{E}}
$$

$\forall t_{q} \in \mathcal{T}_{Q}, \forall t_{d} \in \mathcal{T}_{D}, S A T E S\left(t_{q}, t_{d}\right)$ returns a set of scored approximate tree embeddings for $t_{q}$ in $t_{d}$. The $S A T E S$ function returns a result set, thus capturing 
the possibility of having more than one embedding between a query tree and a document tree.

Intuitively, the $S A T E S$ function states "how well" a data tree $t_{d}$ fits a query tree $t_{q}$, also taking care of multiple fittings. To determine the scored embeddings, the function is defined in a recursive fashion. In order to show the embedding process, we follow an example-driven approach, and we provide the formal definition of the $S A T E S$ function in appendix.

We recall the example of Fig. 2] and we try to find an embedding for $t_{q}$ in $t_{d}$. For simplicity, we start considering only the second branch of the $t_{d}$ tree, i.e. the one relating to "Music store". We refer to it as the current $t_{d}$, and we denote it with $t_{d}^{*}$. Then, we will show later how the final embedding of $t_{q}$ in $t_{d}$ will include also the embedding for the "Artist shop" store, thus resulting in a set of embeddings. Starting top-down, the method tries to find a match between roots' labels. If a match is possible, then the $S A T E S$ function recursively applies to children of $t_{q}$ and $t_{d}^{*}$. This is made according to a bipartite graph matching 1 between the children of $t_{q}$ and the children of $t_{d}^{*}$, and we denote it with $\mathcal{M}_{t_{q}}^{t_{d}}$. In our example, since the labels of $t_{q}$ 's root and $t_{d}^{*}$ 's root match, we consider the most promising matching $\mathcal{M}_{t_{q}}^{t_{d}}$ between the pairs (address $\mathbf{s}_{q}$, address $\mathbf{s}_{d}$ ), and $\left(\mathrm{cd}_{q}, \mathrm{~cd}_{d}\right)$, where indices $q$ and $d$ denote the belonging to query and data trees, respectively. In fact, other possible matchings $\mathcal{M}_{t_{q}}^{t_{d}}$, involving for instance the "Music store" node, do not produce relevant results. However, alternative matchings help in finding structural discrepancies, as we will show later in this Section. Then, the embedding method proceeds recursively on the abovementioned pairs.

First, let us consider the pair (address , address $_{d}$ ): We have that $t_{q}^{*}=$ $\operatorname{supp}\left(\operatorname{addres}_{q}\right)$ and $t_{d}^{*}=\operatorname{supp}\left(\right.$ address $\left._{d}\right)$, with $t_{q}^{*}$ the current $t_{q}$. Since roots' labels match, a $\mathcal{M}_{t_{q}}^{t_{d}}$ is established between nodes city $_{q}$ and "Manhattan, . . " ${ }_{d}$. Then, recursion is applied again. However, in this case no similarity is found for roots' labels (at present, $t_{q}^{*}=\operatorname{supp}\left(\operatorname{city}_{q}\right)$, and $t_{d}^{*}=\operatorname{supp}\left(" M a n h a t t a n, \ldots{ }_{d}\right)$ ). Thus, the method considers two possible strategies:

1. (optimistic): it tries to find a complete embedding for $t_{q}^{*}$ at a deeper level in $t_{d}^{*}$. This means that the search context is changed to a more specific context;

2. (pessimistic): it intentionally gives up looking for a match of $t_{q}^{*}$ s root (thus accepting a partial match), and tries to satisfy the remaining query conditions $\left(t_{q}^{*}=\operatorname{supp}\left(\right.\right.$ "New York" $\left.\left.{ }_{q}\right)\right)$, by proceeding either:

a) in the current data context, i.e. $t_{d}^{*}$ stays unchanged, or

b) changing the context, i.e. looking for a match at a deeper level in $t_{d}^{*}$

In the current example, it is easy to notice that the only feasible strategy is $2 \mathrm{a}$. In fact, since $t_{d}^{*}$ is indeed a leaf, it is not possible to change any search context,

${ }^{1}$ Consider a graph $G=(V, E) . G$ is bipartite if there is a partition $V=A \cup B$ of the nodes of $G$ such that every edge of $G$ has one endpoint in $A$ and one endpoint in $B$. A matching is a subset of the edges no two of which share the same endpoint. In our case $A=\operatorname{children}\left(\operatorname{root}\left(t_{q}\right)\right)$ and $B=\operatorname{children}\left(\operatorname{root}\left(t_{d}^{*}\right)\right)$. 
thus making alternatives 1 and $2 \mathrm{~b}$ not applicable. Then recursion concludes for this branch (i.e. for the pair ( address $_{q}$, address $d$ )), since the current trees are actually two leaves, and a match is found for them.

Now, let us get back to the pair $\left(\mathrm{cd}_{q}, \mathrm{~cd}_{d}\right)$ : We have that their labels match, and two matchings $\mathcal{M}_{t_{q}}^{t_{d}}$ are possible among their children:

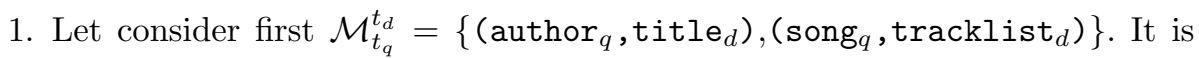
easy to show that the former pair does not produce any result; on the other hand, in the latter one we follow strategy 1, and we recursively compute $S A T E S$ on the pair $\left(\right.$ song $_{q}$, track $\left._{d}\right)$. This time, labels are similar and the embedding process goes on, matching (title $e_{q}$, title $_{d}$ ), since the pair $\left(\text { title }_{q} \text {, author }\right)_{d}$ does not return any result. Finally, the pair ("Love" $q$, "Love of $\ldots{ }_{d}$ ) is added to the embedding.

2. As to the second possible matching $\mathcal{M}_{t_{q}}^{t_{d}}=\left\{\left(\right.\right.$ song $_{q}$, title $\left._{d}\right)$, (author $_{q}$, tracklist $d$ )\}, once again we neglect the former pair. In fact, although an embedding is retrieved, i.e. $\left\{\left(t_{i t l e_{q}}, t_{i t l e_{d}}\right)\right\}$, the corresponding leaves do not match, thus making the embedding not significant 2 With regard to the second pair, the double application of strategy 1 allows for discovering the embedding of (author , author $_{d}$ ), including the pair ("Elton John" $q$, "Elton John" $d$ ). On the other hand, similarly to the previous case, the pair (author $q$, title $_{d}$ ) leads to empty results.

Thus, in order to capture structural unbalances, the SATES function takes into account all possible matchings $\mathcal{M}_{t_{q}}^{t_{d}}$. Finally, for the sake of completeness, recall that the starting embedding was $S A T E S\left(t_{q}, t_{d}\right)$. The set-oriented approach of our method allows for returning two possible solutions, through the double application of strategy 1. The former time to try the embedding of the pair (cdstore ${ }_{q}$, cdstore $_{d}^{\prime}$ ), and the latter one for the pair (cdstore , cdstore $_{d}^{\prime \prime}$ ), where the number of apices denotes the first and second CD store in Doc1, respectively. A further situation where the set-oriented approach contributes in the retrieval of multiple occurrences of results is shown in figures 3 and 4, where the final embedding set is obtained by the union of the embeddings originated by the pairs $\left(\mathrm{cd}_{q}, \mathrm{~cd}_{d}^{\prime}\right)$ and $\left(\mathrm{cd}_{q}, \mathrm{~cd}_{d}^{\prime \prime}\right)$, respectively.

\section{Relevance Computation}

Most approaches 4,7,10,17,20] score results with ranking values that, individually, do not provide information on the query rate satisfied by an answer. Assume, for instance, to compare results coming from two different queries $q_{1}$ and $q_{2}$. According to most scoring methods [417], it is possible that one document that satisfies 1 condition (out of 2) of $q_{1}$ is assigned the same score of a document

\footnotetext{
${ }^{2}$ This policy privileges embeddings with query leaves matching. This is to limit the huge amount of results that satisfy structural constraints, but do not match content conditions. From a strict structural point of view, the above pair should belong to the embedding.
} 
that satisfies 9 conditions (out of 10) of the more complex query $q_{2}$. Although results are incomparable, one would expect documents (exactly) satisfying a high percentage of conditions to score higher than documents (exactly) satisfying a lower rate. Thus, besides information on correctness of results, a measure of completeness is desirable. As to XML documents, this information is somehow made more complex by the presence of structure inside documents. This implies that some knowledge on completeness is supposed to provide also information on the matching rate of query structure. Then, apart from queries where the user explicitly specifies not to take care of the depth where information may be found in, also cohesion of data retrieved is an important element to be considered when ranking results.

Here, we provide a scoring method that captures the above-mentioned features. We start modelling a set of properties for each embedding $\tilde{e}$. Property values are normalized in the interval $[0,1]$, where values close to 1 denote high satisfaction. Properties are:

Semantic Completeness. It is a measure of how much the embedding is semantically complete with respect to the given query. It is computed it as the ratio between the number of query nodes in the embedding, $n_{q}^{\tilde{e}}$, and the total number of query nodes, $n_{q}$ :

$$
\gamma_{1}=\frac{n_{q}^{\tilde{e}}}{n_{q}}
$$

Semantic Correctness. It states how well the embedding satisfies semantic requirements. It represents the overall semantic similarity captured by the nodes in the embedding. This is computed as a combination of label similarities of matching nodes, possibly lowered by type mismatchings (attribute vs. element nodes):

$$
\gamma_{2}=\bigwedge_{q_{i} \in \operatorname{som}(\mathcal{E})} \operatorname{sim}\left(\operatorname{label}\left(q_{i}\right), \operatorname{label}\left(\tilde{e}\left(q_{i}\right)\right)\right)
$$

where $\wedge$ is a scoring function 9 the computes the conjunction of label similarities. For instance, $\wedge$ could be a fuzzy t-norm [13], such as the min function or the product operator.

Structural Completeness. It represents the structural coverage of the query tree. It is computed as the ratio between: 1) the number of node pairs in the image of the embedding, $h p_{q}^{\tilde{e}}$, that satisfy the same hierarchica 3 relationship of the query node pairs which are related to, and 2) the total number of hierarchy-related pairs in the query tree $\left(h p_{q}\right)$ :

$$
\gamma_{3}=\frac{h p_{q}^{\tilde{e}}}{h p_{q}}
$$

Structural Correctness. It is a measure of how many nodes respect structural constraints. It is computed as the complement of the ratio between the

${ }^{3}$ Either parent-child or ancestor-descendant relationship. 
number of structural penalties $p$ (i.e. unbalances) and the total number of hierarchy-related pairs in the data tree that also appear in the embedding:

$$
\gamma_{4}=1-\frac{p}{h p_{d}^{\tilde{e}}}
$$

Cohesion of results. It represents the grade of fragmentation of the resulting embedding. It is computed as the complement of the ratio between the number of intermediate data nodes $i n_{d}^{\tilde{e}}$ among the nodes in the embedding, and the total number of data nodes in the embedding, also including the intermediate ones, $n_{d}^{\tilde{e}}$ :

$$
\gamma_{5}=1-\frac{i n_{d}^{\tilde{e}}}{n_{d}^{\tilde{e}}}
$$

These properties can be naturally partitioned in two sets: properties related to semantics, and properties concerning structure. The combination of the first two scores provides the overall information on semantic satisfaction. As to the remaining properties, they all provide different perspectives for the evaluation of structure similarity. A combination of them indicates a global measure, that summarizes the structural satisfaction of the retrieved data. However, it is beyond the scope of this paper to evaluate which is the best function to be used for combining these scores in the computation of the overall score $\sigma$ of an embedding, as defined in Def. 2. Clearly, additional flexibility can be reached by assigning weigths to each $\gamma_{i}$ to denote the different importance of each property.

\subsection{Comparison with Related Approaches}

Table1 1 shows a comparison of our ranking method with other similar approaches, according to different relaxations on structure.

Table 1. Relaxations supported on structure

\begin{tabular}{|c||c|c|c|c|}
\hline Approach & partial match & unbalance & intermed. data nodes & multiple occurr. \\
\hline \hline XXL [20] & no & no & yes & no \\
\hline XIRQL [10] & no & no & yes & no \\
\hline Damiani et al. [7] & no & no & yes & no \\
\hline Amer-Yahia et al. [4] & no & yes & yes & no \\
\hline ApproXQL [17] & yes & no & yes & no \\
\hline SATES & yes & yes & yes & yes \\
\hline
\end{tabular}

All the discussed methods allow for intermediate data nodes in the results. However, all but ApproXQL do not consider the number of exceeding data nodes in the computation of scores. In fact, all methods except ApproXQL would return

${ }^{4}$ Subtree Promotion in 4 captures the unbalances described in Def. 1 point $b$ ). The symmetric case, point $c$ ), is not discussed. 
the same score for the approximate tree embeddings of figures 4 and 5 , even though the former embedding presents a higher fragmentation 5

However, consider the query embedding of Fig. 6, where the current query is simplified with respect to the sample we used throughout the paper, since it does not require any constraint on CD store location.

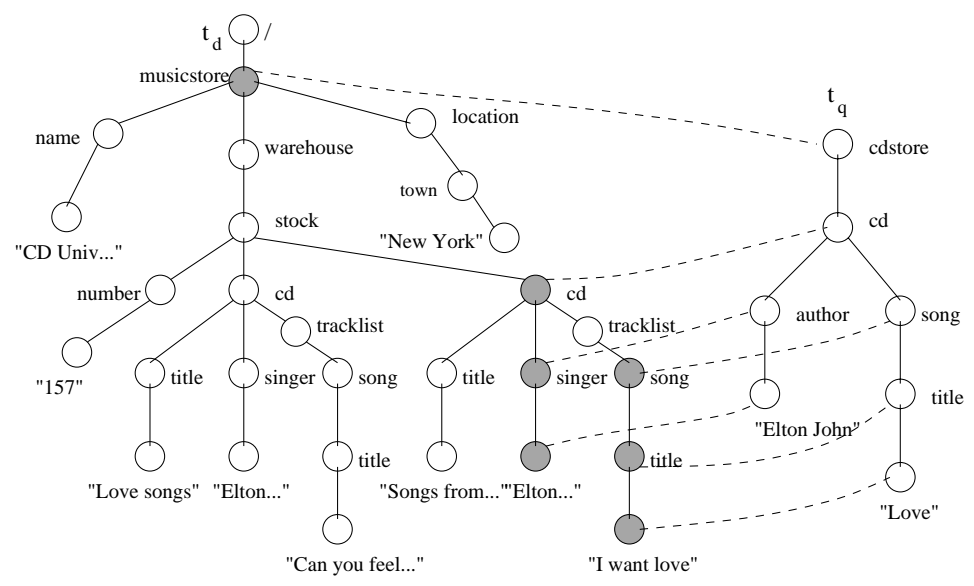

Fig. 6. Embedding of the simplified sample query

Table 2 shows how ApproXQL and $S A T E S$ evaluate the embeddings of figures 4 and 6 For ApproXQL, the same score would be assigned in both cases, since the

Table 2. Different scoring in presence of different cohesion of data

\begin{tabular}{|c||c|c|}
\hline Approach & Score for embedding of Fig. 4 & Score for embedding of Fig. 6 \\
\hline \hline ApproXQL & high & high \\
\hline$S A T E S$ & high & medium \\
\hline
\end{tabular}

number of relaxations to meet data organization can be quantified in the same number of intermediate nodes for both queries (3 nodes, indeed). Nevertheless, the set of query requirements is different, and the number of exceeding nodes is proportionally more heavy for the embedding of Fig. 6. SATES takes into account this feature, and assigns two different scores to results, scoring higher the embedding of Fig. 4 .

${ }^{5}$ For simplicity, we assume the matchings on labels to score the same for both embeddings, so that only evaluation on structure affects the ranking. 


\section{Implementation}

We implemented a prototype and experienced our similarity measure on a real collection of XML documents. The dataset used is provided by the Astronomical Data Center [1]. The prototype has been developed in Java2 v.1.3.1 and uses the API for XML parsing, and Xerces 1.4.4 to parse documents. As to semantic similarity, the system refers to the WordNet semantic network, 2] exploiting relationships like synonymy, hyperonymy, and holonymy among terms, and accesses it through the Java WordNet Library (JWNL). As a similarity measure we used an adaptation of the Sussna's formula [5]. We plan to experience and refine our method, in order to find the better rules to be used for our combine and ranking functions, with the aim of representing results at the best of their meaning.

\section{Conclusions and Future Work}

Most of the existing ranking approaches are inadequate when querying heterogeneous collections of XML documents. In fact, as to similarity on documents' structure, they basically require all query conditions are somehow preserved in the data retrieved. Also, they do not fully exploit relaxations on structure to capture slightly different organization of data.

We presented an approach that widens the spectrum of relevant data, including solutions that also partially satisfy query requirements, and that approximate text organization inside documents, also capturing unbalances of structure and multiple occurrences of query conditions. Then, we rank results according to a set of properties of the retrieved data. These properties provide, besides correctness, a measure of completeness of query satisfaction, as well as knowledge about cohesion of results. In order to augment query flexibility we plan to allow the user to express preferences on either the semantics or the structure of a query. As to queries with conditions referring to ordered data, we intend to study constraints on our generic unordered tree embedding method. We are aware of the problem of blindly querying XML data: Documents might be organized largely differently from the user's point of view. Thus, we plan to study new complex hierarchical relaxations on data, and to make this process transparent to the user. With regard to implementation, in order to cope with the possible hugeness of approximate results returned, we plan to use threshold conditions, to keep only the most relevant results.

\section{References}

1. ADC XML Resources Home Page. http://xml.gsfc.nasa.gov/.

2. WordNet Home Page. http://www.cogsci.princeton.edu/ wn/.

3. XML Information Set. http://www.w3.org/TR/xml-infoset.

4. S. Amer-Yahia, S. Cho, and D. Srivastava. Tree Pattern Relaxation. In Proc. of the 8th Int. Conf. on Extending Database Technology (EDBT 2002), March 2002. 
5. A. Budanitsky. Lexical Semantic Relatedness and its Application in Natural Language Processing. Technical Report CSRG-390, University of Toronto, 1999.

6. S. Ceri, S. Comai, E. Damiani, P. Fraternali, S. Paraboschi, and L. Tanca. XMLGL: A Graphical Language for Querying and Restructuring XML Documents. Computer Networks, 31:1171-1187, 1999.

7. E. Damiani and L. Tanca. Blind Queries to XML Data. In In Proc. of Int. Conf. on Database and Expert Systems Applications (DEXA), pages 345-356, 2000.

8. A. Deutsch, M. Fernandez, D. Florescu, A. Levy, and D. Suciu. XML-QL: A Query Language for XML. In Proc. Int. World Wide Web Conference), Canada, 1999.

9. R. Fagin. Combining Fuzzy Information from Multiple Systems. In Proceedings of the 15th ACM Symposium on Principles of Database Systems (PODS'96), pages 216-226, Montreal, Canada, June 1996.

10. N. Fuhr and K. Großjohann. XIRQL: A Query Language for Information Retrieval in XML Documents. In Proc. ACM SIGIR Conference, 2001.

11. R. Goldman, J. McHugh, and J. Widom. From Semistructured Data to XML: Migrating the Lore Data Model and Query Language. In In Proc. of 2nd Int. Workshop on the Web and Databases (WebDB'99), Philadelphia, PA, June 1999.

12. P. Kilpeläinen. Tree Matching Problems with Application to Structured Text Databases. PhD thesis, Dept. of Computer Science, Univ. of Helsinki, SF, 1992.

13. G.J. Klir and B. Yuan. Fuzzy Sets and Fuzzy Logic. Prentice Hall PTR, 1995.

14. J. Madhavan, P. A. Bernstein, and E. Rahm. Generic Schema Matching with Cupid. In Proc. of the 27th VLDB Conf., pages 49-58, Rome, Italy, 2001.

15. S. Melnik, H. Garcia-Molina, and E. Rahm. Similarity Flooding: A Versatile Graph Matching Algorithm and its Application to Schema Matching. In Proc. of the 18th Int. Conf. on Data Engineering (ICDE 2002), San Jose, CA, March 2002.

16. J. Robie, L. Lapp, and D. Schach. XML Query Language (XQL). In Proc. of the Query Language Workshop (QL'98), Cambridge, Mass., 1998.

17. T. Schlieder. Similarity Search in XML Data Using Cost-Based Query Transformations. In Proc. of 4th Int. Work. on the Web and Databases (WebDB01), 2001.

18. T. Schlieder and H. Meuss. Result Ranking for Structured Queries against XML Documents. In DELOS Workshop: Information Seeking, Searching and Querying in Digital Libraries, 2000.

19. T. Schlieder and F. Naumann. Approximate Tree Embedding for Querying XML Data. In In Proc. ACM SIGIR Workshop on XML and Information Retrieval, Athens, Greece, July 2000.

20. A. Theobald and G. Weikum. Adding Relevance to XML. In Proc. 3rd Int. Workshop on the Web and Databases (WebDB 2000), pages 35-40, May 2000.

21. J. Wolff, H. Florke, and A. Cremers. Searching and Browsing Collections of Structural Information. In Proc. of the IEEE Advances in Digital Libraries, pages 141150, USA, May 2000.

\section{A The $S A T E S$ Function}

For simplicity, let $\operatorname{sim}\left(t_{q}, t_{d}\right)$ be $\operatorname{sim}\left(\operatorname{label}\left(\operatorname{root}\left(t_{q}\right)\right)\right.$, label $\left.\left(\operatorname{root}\left(t_{d}\right)\right)\right)$, and let $s\left(t_{q}, t_{d}\right)=\rho\left(t_{q}, t_{d},\left\{\operatorname{root}\left(t_{q}\right), \operatorname{root}\left(t_{d}\right)\right\}\right)$. We use $\mathcal{M}$ in place of $\mathcal{M}_{t_{q}}^{t_{d}}$. The $\ominus_{q}$ and $\ominus_{d}$ functions change the scores of a set of approximate tree embeddings, according to a lowering factor. New scores capture the unsuccessful match of a query node and a data node, respectively. The $\otimes$ function generates a new score from a set of $n$ given scores in $\mathcal{S}$. 


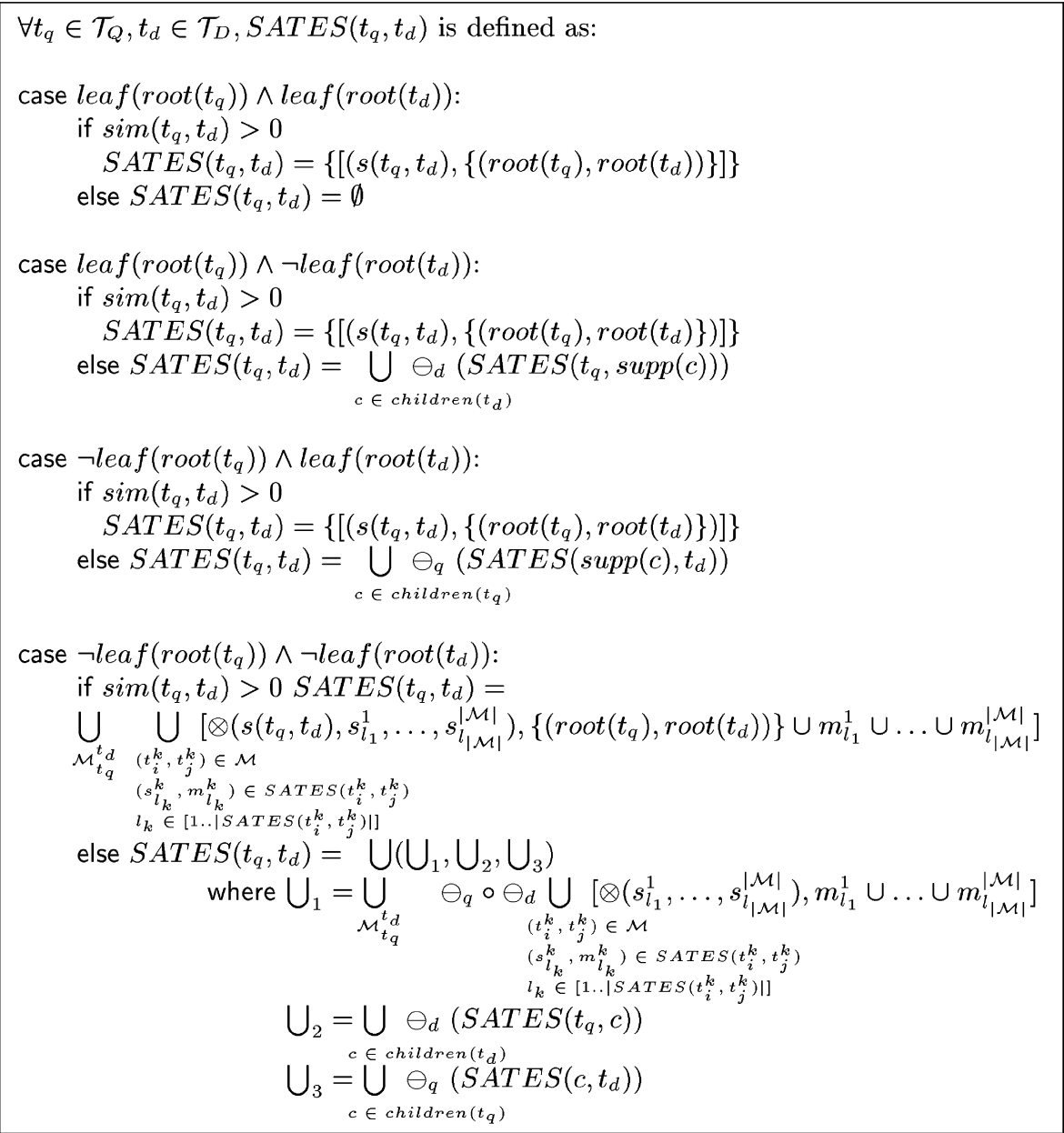

